\title{
FERMION PROPAGATORS ON A FOUR-DIMENSIONAL RANDOM-BLOCK LATTICE
}

\author{
Ting-Wai CHIU ${ }^{1}$ \\ Physics Department, 206-49, California Institute of Technology, Pasadena, CA 91125, USA
}

Received 29 January 1988

\begin{abstract}
Fermion propagators, composite boson propagators and the fermion condensate are calculated numerically on the four-dimensional random-block lattice, respectively. The ensemble-averaged fermion propagator agrees with the continuum propagator for distances greater than three average lattice spacings. The results on the fermion condensate show that the chiral symmetry of the doubled modes is broken in the continuum limit. The Goldstone boson arising from the broken symmetry is revealed by examining the composite pseudo-scalar propagator. The doubled fermion and the Goldstone boson both acquire masses of the order of inverse lattice spacing and thus decouple from the theory in the continuum limit.
\end{abstract}

Recently, the properties of the Dirac fermion have been studied on two-dimensional and four-dimensional random lattices [1], respectively. It has been shown that the random lattice may provide a natural way to resolve the fermion doubling problem. The chiral symmetry of the doubled modes (at the tree level) is spontaneously broken in the random lattice. The fermions associated with the doubled modes acquire masses of the order of the inverse of the average lattice spacing $a$, and the approximate Goldstone boson arising from the broken symmetry has a mass of the order $\sqrt{m / a}$, where $m$ is the mass of the primary fermion. In the continuum limit they become infinitely heavy and therefore decouple from the theory. The effects of the doubled fermions extend only a few lattice spacings.

In this paper we investigate the properties of the Dirac fermion on the four-dimensional random-block lattice [2]. A formulation of the gauge theory on a random-block lattice will be presented elsewhere [3]. The sites of a random-block lattice are the cartesian product of the set of randomized coordinates from each dimension. An example of a two-dimensional random-block lattice is shown in fig. 1 . Unlike the

This research is partially supported by US Department of Energy Grant No. DE-FG03-85ER25009, and by National Science Council, R.O.C.

I On leave of absence from Department of Physics, National Taiwan University, Taipei, Taiwan 10764, R.O.C.

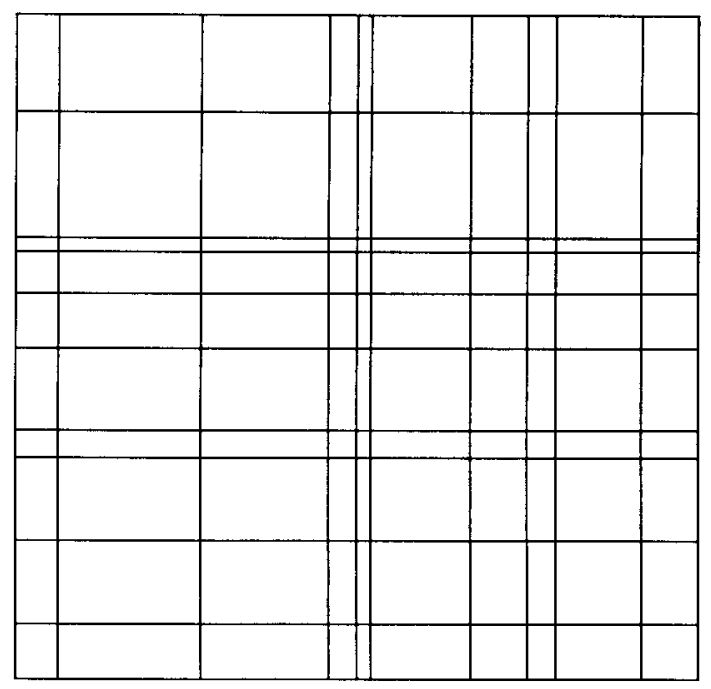

Fig. 1. An example of a two-dimensional random-block lattice.

random lattice, there are preferred directions in a random-block lattice. The rotational invariance can only be restored in the continuum limit. If the randomness of a random lattice could provide the breaking of the chiral symmetry of the doubled modes, then we would anticipate that the same should also happen in a random-block lattice. The results in this paper confirmed our expectations. Furthermore, field theoretic calculations on a 4D random-block lattice 
are much simpler than their counterparts on a 4D random lattice. This allows us to perform the calculations on larger lattices and obtain new results which are difficult to obtain on a 4D random lattice. The fermion propagators are obtained by averaging over 1000 random-block lattices, each with $20 \times 20 \times 20 \times 20$ sites. It agrees with the continuum limit excellently for distances greater than three average lattice spacings, in agreement with earlier results [4] in a four-dimensional random lattice. The fermion condensates $\langle\bar{\psi} \psi\rangle$ are calculated on $4 \mathrm{D}$ random-block lattices of 1296,4096 and 10000 sites, respectively. Our results show that the chiral symmetry of the doubled modes is spontaneously broken in the continuum limit. The existence of the Goldstone boson arising from this broken symmetry can be revealed by examining the composite pseudo-scalar propagator. The composite scalar and pseudo-scalar propagators are obtained by averaging over 1000 random-block lattices, each with $10 \times 10 \times 10 \times 10$ sites. The short-distance effects of the Goldstone boson are seen explicitly in the composite pseudo-scalar propagator.

On a four-dimensional random-block lattice the Dirac fermion action is [3]

$$
\begin{aligned}
A_{\mathrm{L}} & =\sum_{i} \sum_{\mu} \omega_{i} \bar{\psi}_{i} \gamma^{\mu} K_{i}^{\mu}\left(\psi_{i+\mu}-\psi_{i-\mu}\right)+m \sum_{i} \omega_{i} \bar{\psi}_{i} \psi_{i} \\
& \equiv \bar{\Psi}\left(\gamma^{\mu} T^{\mu}+m W\right) \Psi,
\end{aligned}
$$

where $\bar{\psi}_{i}$ and $\psi_{i}$ are two independent four-component spinors at site $i, \omega_{i}$ is the weight of the site $i, K_{l}^{\mu}$ is the inverse of the distance between the sites $i+\mu$ and $i-\mu, m$ is the mass and the $\gamma^{\mu}$ are the Dirac matrices. In terms of the coordinates of neighboring sites, $\omega_{i}$ and $K_{i}^{\mu}$ can be written as

$$
\begin{aligned}
& \omega_{i}=\frac{1}{16}\left(x_{i 1+1}^{1}-x_{i_{1}-1}^{1}\right)\left(x_{i 2+1}^{2}-x_{i 2-1}^{2}\right) \\
& \quad \times\left(x_{i 3+1}^{3}-x_{i 3-1}^{3}\right)\left(x_{i 4+1}^{4}-x_{i 4-1}^{4}\right), \\
& K_{i}^{\mu}=\left(x_{i+\mu}-x_{i-\mu}\right)^{-1} .
\end{aligned}
$$

The Dirac matrices are in the chiral representation,

$$
\gamma_{j}=\left(\begin{array}{cc}
0 & -\mathrm{i} \sigma_{j} \\
\mathrm{i} \sigma_{j} & 0
\end{array}\right), \quad j=1,2,3,
$$

$\gamma_{4}=\left(\begin{array}{ll}0 & I \\ I & 0\end{array}\right)$, $\gamma_{5}=\gamma_{1} \gamma_{2} \gamma_{3} \gamma_{4}=\left(\begin{array}{rr}I & 0 \\ 0 & -I\end{array}\right)$,

where $\sigma_{j}$ are Pauli matrices and $I$ is the $2 \times 2$ unit matrix. In the continuum limit, (1) goes to the continuum Dirac fermion action

$A_{\mathrm{c}}=\int \mathrm{d}^{4} x \bar{\psi}(x)\left(\gamma^{\mu} \partial^{\mu}+m\right) \psi(x)$.

The continuum Dirac fermion propagator $S_{\mathrm{F}}(x)$ satisfies the equation

$\left(\gamma^{\mu} \mathrm{\partial}^{\mu}+m\right) S_{\mathrm{F}}(x)=\delta^{4}(x)$.

The inversion of the operator $\left(\gamma^{\mu} \partial^{\mu}+m\right)$ can be carried out more effectively by using the following simple identity

$\left(\gamma^{\mu} \partial^{\mu}+m\right)^{-1}=\left(m-\gamma^{\mu} \partial^{\mu}\right)\left(m^{2}-\partial^{2}\right)^{-1}$.

The significance of this identity is that we can reduce the task of inverting a complex matrix of dimension $4 \times(L \times L \times L \times L)^{2}$ to one of inverting a real symmetric matrix of dimension $(L \times L \times L \times L)^{2}$. In the continuum limit, $S_{\mathrm{F}}(x)$ can be calculated analytically and the result is

$$
\begin{aligned}
& S_{\mathrm{F}}(x)=\left(m-\gamma_{\mu} \partial_{\mu}\right)\left\langle x\left|\left(m^{2}-\partial^{2}\right)^{-1}\right| 0\right\rangle \\
& \quad=(m / 2 \pi)^{2}\left[x^{-1} K_{1}(m x)+\gamma_{\mu} x_{\mu} x^{-2} K_{2}(m x)\right] \\
& \quad \equiv S_{0}(x) \mathbf{1}+\gamma_{\mu} S_{\mu}(x),
\end{aligned}
$$

where $K_{1}(m x)$ and $K_{2}(m x)$ are the modified Bessel functions of the second kind.

In a finite-size box of dimension $L \times L \times L \times L$ with periodic boundary conditions, the above continuum results must be modified by adding the contributions due to the image sources. This summation in general requires a fair amount of computational resources. However, in the cases we have studied, summing 20 image sources in each direction is already accurate enough for our purposes.

The fermion propagator in a random-block lattice is the inverse of the matrix $\left(\gamma^{\mu} T^{\mu}+m W\right)$ defined in (1). The $W$ matrix is a diagonal matrix of the site weights, while the $T$ matrix corresponds to the momentum operator. The matrix inversion was carried out by using the simple identity (4).

$$
\begin{aligned}
S_{i j}^{\mathrm{L}} & =\left\langle\psi_{i} \bar{\psi}_{j}\right\rangle=\left(\gamma_{\mu} T_{\mu}+m W\right)_{i j}^{-1} \\
& =\left(m W-\gamma_{\mu} T_{\mu}\right)_{i k}\left(m^{2} W^{2}-T^{2}\right)_{k j}^{-1} \\
& =\left(S_{0}^{\mathrm{L}}\right)_{i j}+\gamma^{\mu(}\left(S_{\mu}^{\mathrm{L}}\right)_{i j} .
\end{aligned}
$$


The real symmetric matrix $\left(m^{2} W^{2}-T^{2}\right)$ was inverted by the conjugate gradient method. The ensemble-averaged fermion propagator was calculated by generating 1000 random-block lattices, each of $20 \times 20 \times 20 \times 20$ sites with the coordinates of two sites fixed, and by taking the arithmetic average over the random-block lattices. The fixed sites corresponding to the end-points of the propagator are chosen to be on the body diagonal such that the ensembleaveraged $S_{\mu}(x)$ should be nearly the same for all $\mu$. Another advantage of this choice is that the summation of the image sources in the continuum solution is much simpler for the symmetric configurations. The results of ensemble average $\left\langle S_{0}^{\mathrm{L}}(x)\right\rangle$ in comparison with the continuum results are plotted in fig. $2 \mathrm{a}$. The average of $\left\langle S_{\mu}^{\mathbf{L}}(x)\right\rangle$ over all $\mu$ are plotted in fig. $2 \mathrm{~b}$. The average lattice spacing is one and the dimension of the lattice is $20 \times 20 \times 20 \times 20$. The agreement between the ensemble-averaged propagator and the continuum propagator is excellent for distances greater than three average lattice spacings.

The fermion propagator on a random-block lattice is the same as the continuum propagator at large distances suggests that the doubled modes only have effects at distances of the order of the lattice spacing $a$. Therefore the doubled fermions might have acquired masses of order $1 / a$, similar to what has found in the two-dimensional random lattice [5]. If this scenario is correct, then the mass of the doubled fermion would survive in the limit $m a \rightarrow 0$, and the chiral symmetry of the doubled fermion must be broken spontaneously. This can be verified explicitly by examining the fermion condensate. The fermion condensate is

$$
\begin{aligned}
& \langle\bar{\psi} \psi\rangle=\frac{1}{L^{4}} \sum_{i} \omega_{i}\left\langle\bar{\psi}_{i} \psi_{i}\right\rangle \\
& =\frac{1}{L^{4}} \sum_{i} \omega_{i} \operatorname{tr}\left(\gamma^{\mu} T^{\mu}+m W\right)_{i i}^{-1} .
\end{aligned}
$$

This expression was evaluated numerically on several different $4 \mathrm{D}$ random-block lattices up to $10 \times 10 \times 10 \times 10$ sites with periodic boundary conditions. The results are shown in fig. 3 . The rising and falling of the condensates as $m \rightarrow 0$ are only due to the finite size effects. When the Compton wavelengths of the particles contributing to $\langle\bar{\psi} \psi\rangle$ become of the order or greater than the lattice size $L$ (i.e. $m L \lesssim 1$ ), $\langle\bar{\psi} \psi\rangle$ starts to rise. However a finite volume system
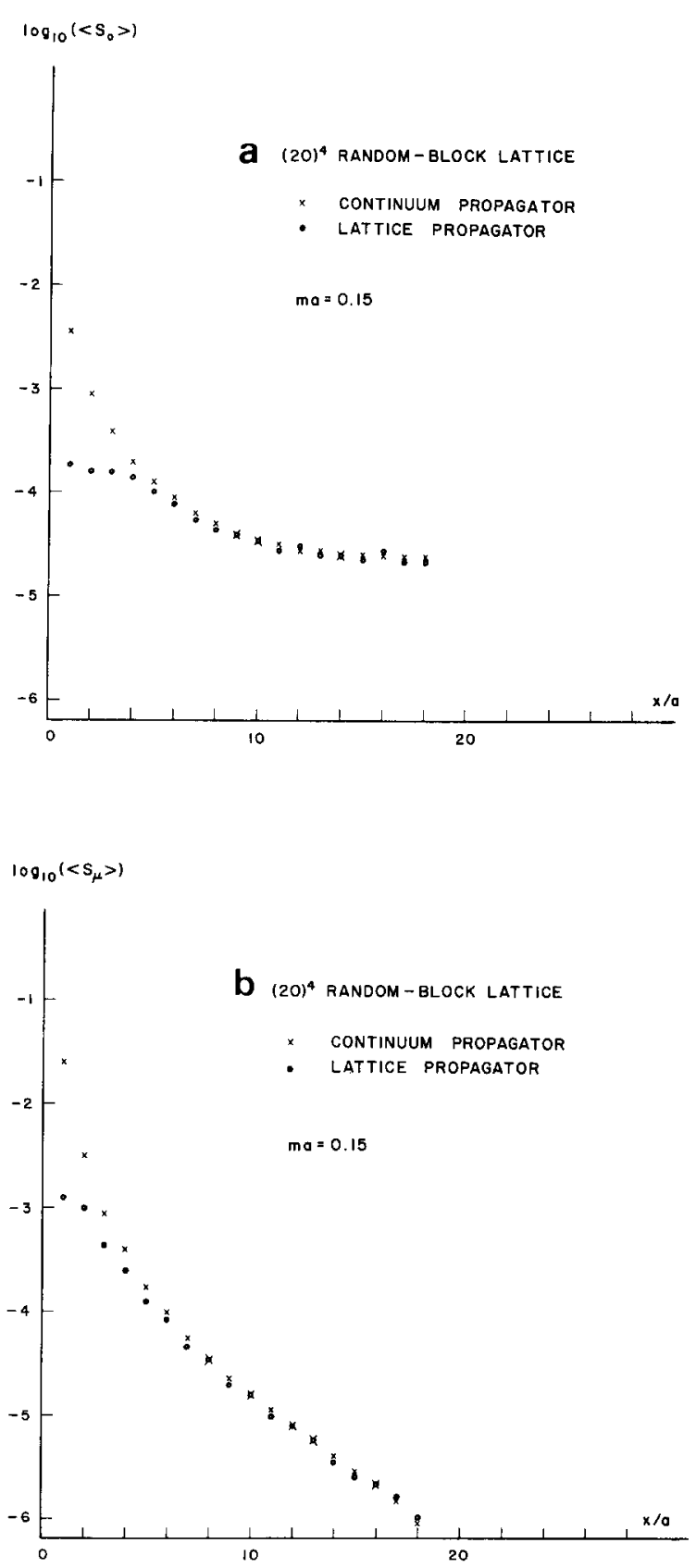

Fig. 2. Ensemble average of the fermion propagator over 1000 four-dimensional random-block lattices of $20 \times 20 \times 20 \times 20$ sites with periodic boundary conditions and with $m a=0.15$. The crosses are the ensemble-averaged propagator while the circles are the continuum limits with the same boundary conditions and $m a=0.15$. (a) The $S_{0}$ component of the fermion propagator. (b) The average of the $S_{\mu}$ components of the fermion propagator. 


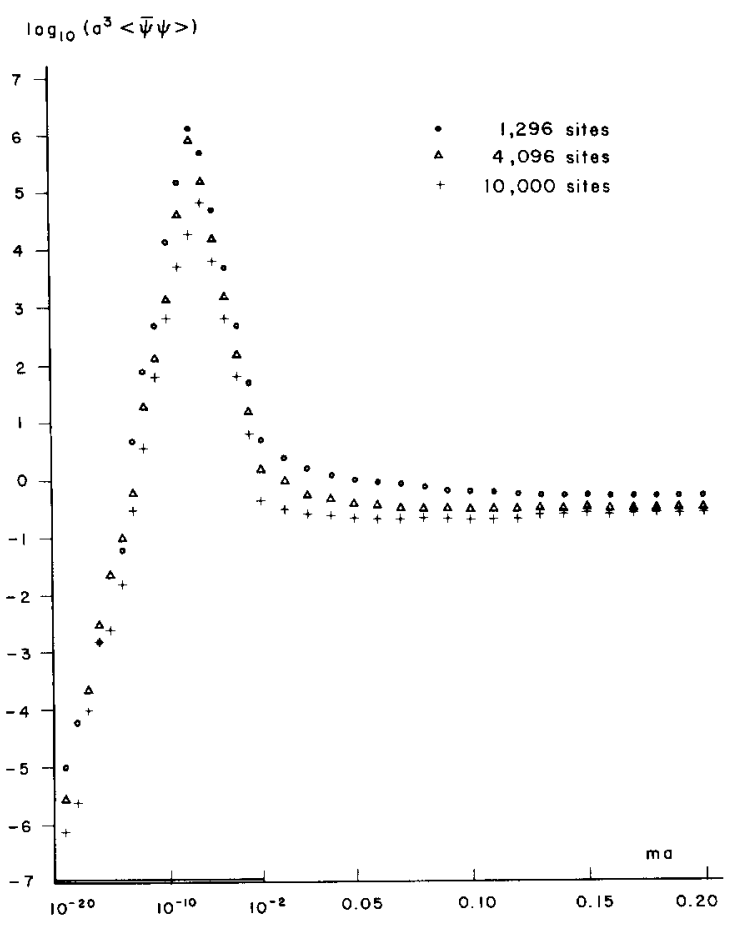

Fig. 3. Fermion condensate $\langle\bar{\psi} \psi\rangle$ versus mass for four-dimensional random-block lattices of 1296,4096 and 10000 sites with periodic boundary conditions. The scale of the horizontal axis is not linear; from $10^{-20}$ to $10^{-2}$ is logarithmic while from 0.01 to 0.20 is linear. This figure provides evidence that in the continuum limit chiral symmetry is spontaneously broken for the Dirac theory on a 4D random-block lattice.

cannot have true symmetry breaking and therefore as $m \rightarrow 0$, the condensate must vanish. On larger lattices the rising points go to smaller values of $m$ and the heights of the peaks decrease. This is seen clearly by comparing the curves of 10000 sites and 1296 sites, respectively. The continuum limit is obtained by first taking the infinite volume limit $L \rightarrow \infty$, and then letting $m a \rightarrow 0$. By taking the continuum limit in fig. 3 , we obtain the fermion condensate to be $\langle\bar{\psi} \psi\rangle \sim 0.5 a^{-3}$ as $m a \rightarrow 0$. Comparing to the fermion condensate $\langle\bar{\psi} \psi\rangle \sim 1.4 a^{-1}$ in the $2 \mathrm{D}$ random lattice [5], the chiral symmetry breaking effect in the random-block lattice is weaker. This can be understood in terms of the degrees of randomness which is equivalent to the "strong force" that breaks the chiral symmetry of the doubled modes. The random-block lattice has preferred directions and therefore has less degrees of randomness.
Associated with this chiral symmetry breaking, there must be a Goldstone boson containing doubled fermions. Since the Goldstone boson is a pseudo-scalar, its presence could be revealed by examining the composite pseudo-scalar propagator. In continuum the composite boson propagator is defined to be

$G_{A B}=\operatorname{tr}\left[A S_{\mathrm{F}}(x) B S_{\mathrm{F}}(-x)\right]$,

where $A$ and $B$ are operators. For $A=B=1, G_{11}$ is a composite scalar propagator. For $A=B=\gamma_{5}, G_{55}$ is a composite pseudo-scalar propagator. On a lattice, the corresponding composite boson propagator from the sites $(i, k)$ to the $\operatorname{sites}(j, l)$ is

$$
\begin{aligned}
\left(G_{A B}^{\mathrm{L}}\right)_{i k, j l} & =-\left\langle\bar{\psi}_{k} A \psi_{i} \bar{\psi}_{j} B \psi_{l}\right\rangle \\
& =\operatorname{tr}\left[A\left(S_{\mathrm{F}}^{\mathrm{L}}\right)_{i j} B\left(S_{\mathrm{F}}^{\mathrm{L}}\right)_{l k}\right] .
\end{aligned}
$$

If $i=k$ and $j=l$, then there is no point-splitting. In terms of the components of the fermion propagator in (6), the composite boson propagators $G_{11}$ and $G_{55}$ can be written as

$$
\begin{aligned}
& \left(G_{11}^{\mathrm{L}}\right)_{i k, j l}=4\left(\left(S_{0}^{\mathrm{L}}\right)_{i j}\left(S_{0}^{\mathrm{L}}\right)_{l k}+\sum_{\mu}\left(S_{\mu}^{\mathrm{L}}\right)_{i j}\left(S_{\mu}^{\mathrm{L}}\right)_{l k}\right), \\
& \left(G_{55}^{\mathrm{L}}\right)_{i k, j l}=4\left(\left(S_{0}^{\mathrm{L}}\right)_{i j}\left(S_{0}^{\mathrm{L}}\right)_{l k}+\sum_{\mu}\left(S_{\mu}^{\mathrm{L}}\right)_{i j}\left(S_{\mu}^{\mathrm{L}}\right)_{l k}\right) .
\end{aligned}
$$

To obtain the composite-boson propagators on the random-block lattice, we average over 1000 randomblock lattices, each of $10 \times 10 \times 10 \times 10$ sites and with four sites fixed. In the case without point-splitting, we choose the four fixed sites to be on the body diagonal, while in the case of point-splitting, two of the fixed sites are off but near the body diagonal. The composite scalar propagators are plotted in fig. 4a. The ensemble-averaged composite scalar propagator with and without point-splitting both agree with the continuum propagator for distances greater than 1.5 average lattice spacings. For distances less than 4 lattice spacings the dominant contribution to the propagators comes from the exchange of a fermion-antifermion pair. For distances beyond 4 lattice spacings the finite-size effects (i.e. the contribution due to a single fermion which propagates from the site $i$ to the site $j$ and then goes around the boundary and back to the site $i$ ) dominate and the propagator becomes a 

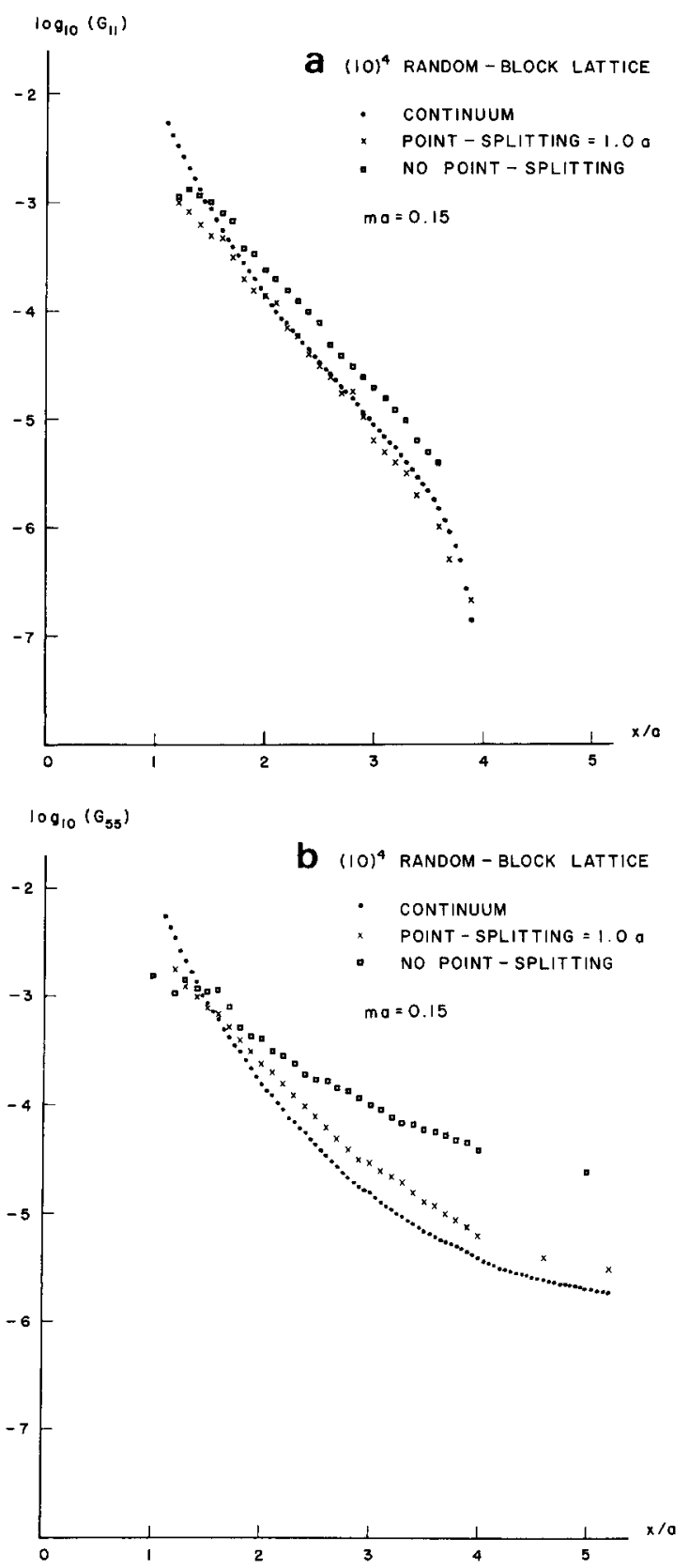

Fig. 4. The composite boson propagators. The propagators are averaged over $10004 \mathrm{D}$ random-block lattices of $10 \times 10 \times 10 \times 10$ sites with periodic boundary conditions and with $m a=0.15$. The crosses are the ensemble averages with point-splitting equal to one average lattice spacing. The squares are ensemble averages without point-splitting. The circles are the continuum propagators with the same boundary conditions and with $m a=0.15$. (a) The composite scalar propagator. (b) The composite pseudoscalar propagator. constant. Therefore we only display the composite propagators up to a distance of 5 lattice spacings in fig. 4. The composite pseudo-scalar propagator is plotted in fig. $4 \mathrm{~b}$. The ensemble-averaged pseudoscalar propagator with point-splitting equal to one lattice spacing agrees with the continuum propagator, while the one without point-splitting differs from the continuum propagator. This is strong evidence of the existence of the Goldstone boson which is the lightest pseudo-scalar bound state of the doubled modes, arising from the broken chiral symmetry. The mass of the Goldstone boson can be obtained by plotting the difference between the propagators with and without point-splitting. Fig. 5 shows that the differences can be fitted by the almost straight line portion of a hyperbolic cosine curve, which is due to a single energy state, the Goldstone boson. The Goldstone mass is estimated to be $\sim 0.61 / a$. On the other hand, current algebra [6] would predict the Goldstone mass to be

$M \sim m^{1 / 2}\langle\bar{\psi} \psi\rangle^{1 / 6}$,

where $m$ is the fermion mass defined in action (1). Using the fermion condensate $\langle\bar{\psi} \psi\rangle \sim 0.5 a^{-3}$ extracted in fig. 3, we obtain the Goldstone mass to be $\sim 0.35 / a$, in agreement with the Goldstone mass

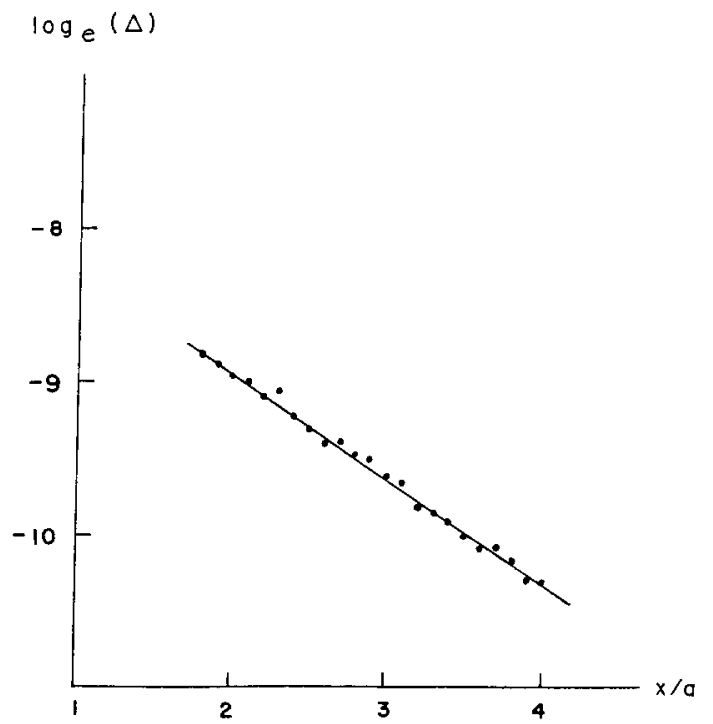

Fig. 5. The difference between the pseudo-scalar propagators with and without point-splitting versus the distances in units of average lattice spacing. The propagators are taken from fig. $4 \mathrm{~b}$. 
$0.61 / a$ shown in fig. 5. This has important implications. First, we now have a self-consistent picture of the doubled sector of the fermion on a $4 \mathrm{D}$ randomblock lattice, which is analogous to the PCAC picture of QCD. The scale of this "strong interaction" in a 4D random-block lattice is $A \sim 0.8 / a$. This "strong interaction" breaks the chiral symmetry of the doubled sector with a condensate of the order $\langle\bar{\psi} \psi\rangle \sim A^{3}$. The Goldstone boson arising from this broken symmetry has a mass of order $\sim \sqrt{m / a}$ which is the lightest bound state of the doubled fermions. The doubled fermions inside the Goldstone boson are confined within a region of order $A^{-1}$ in radius. Second, the ratio of the mass $M$ of the Goldstone boson to the mass $m$ of the primary fermion is $\sim(m a)^{-1 / 2}$, which goes to infinity in the continuum limit. Therefore the Goldstone boson decouples from the theory.

So far, we have restricted ourselves to free fermion theory. In realistic models, one must introduce dynamical gauge fields as link variables. Recent studies [7] of interacting fermions through a Yukawa coupling (without dynamical gauge fields) on a 2D random lattice in weak coupling perturbation theory have shown that the ultraviolet finite diagrams and finite parts of ultraviolet divergent diagrams have the correct continuum limits, while the ultraviolet divergent parts which receive contributions from the doubled fermions diverge as the average lattice spacing goes to zero and thus create a fine tuning problem. It is not certain to what extent that the same would hold for the dynamical gauge models. Since gauge theories on a 4D random-block lattice are much simpler to study than their counterparts on a $4 \mathrm{D}$ random lattice, it would be more feasible to tackle these problems on a 4D random-block lattice.

There are other models in the literature which involve randomness of other kinds. In particular, the random coupling, non-hermitian model of Weingarten and Velikson [8]. The possibility that the WV model has a satisfactory continuum limit has been discussed by several authors [9]. If the nonhermitian effects of the WV model can be confined to the order of lattice spacing, then the WV model might avoid doubling in a similar way to the random lattice and the random-block lattice. At this point, we would like to point out that action (1) can be mapped into a 4D regular lattice action with random couplings. However, our random coupling model is quite different from the WV-type models. First, it is a hermitian model. Second, the positive couplings $2 \omega_{i} K_{i}^{\mu}$ between neighboring spinors are the product of three independent Poisson distributions, each has the mean value equal to the average lattice spacing. These random couplings play an important role in breaking the chiral symmetry of the doubled modes. Since they are space-time dependent, we could visualize them as "link variables" which provide a special short-range strong interaction that only breaks the chiral symmetry of the doubled fermion and confines the effects of the doubled modes to the order of the average lattice spacing. In the regular lattice limit, the random couplings become a constant one and thus this special strong interaction vanishes and the chiral symmetry of the doubled modes is restored. It would be difficult for us to construct such a random coupling model without knowing the random-block lattice in the first place. Nevertheless, the possibility that other hermitian or non-hermitian models could have the correct continuum limit has not been excluded.

Recently we have extended our calculations to chiral fermions on 2D and 4D random-block lattices, respectively. The ensemble-averaged chiral fermion propagators agree with their continuum limits, respectively, for distances greater than three average lattice spacings. The details of these investigations will be presented elsewhere [10].

Finally we would like to remark that all existing lattice QCD or electroweak theory simulation programs on the regular lattice can be converted to those on the random-block lattice without much programming efforts. This is also true for the lattice QCD programs running in the MIMD (multiple instructions / multiple data streams) parallel computers (e.g. the hypercube concurrent computers). We believe that the same does not hold for the random lattice. We are now beginning to convert the Caltech lattice QCD codes to accommodate the random-block lattice.

It is a pleasure to thank Professor Geoffrey C. Fox for his support and kind hospitality extended to me during my stay in the $\mathrm{C}^{3} \mathrm{P}$ group at CALTECH. This research was partially supported by US Department of Energy Grant No. DE-FG03-85ER25009, and by National Science Council, R.O.C. 


\section{References}

[ 1 ] D. Espriu, M. Gross, P. Rakow and J. Wheater, Nucl. Phys. B 275 (1986) 39; Prog. Theor. Phys. Suppl. No. 86 (1986) 304;

R. Friedberg, T.D. Lee and H.C. Ren, Prog. Theor. Phys. Suppl. No. 86 (1986) 322;

Y. Pang and H.C. Ren, Phys. Lett. B 172 (1986) 392; B 195 (1987) 223.

[2] T.W. Chiu, Fermion spectrum on the random lattices, contributed paper XXIII Intern. Conf. on High energy physics (Berkeley, 1986).

[3] T.W. Chiu, Gauge theories on a random-block lattice, in preparation.

[4] Y. Pang and H.C. Ren, Phys. Lett. B 195 (1987) 223.

[5] D. Espriu, M. Gross, P. Rakow and J. Wheater, Nucl. Phys. B 275 (1986) 39.
[6] S.B. Treiman, R. Jackiw and D.J. Gross, Lectures on Current algebra and its applications (Princeton U.P., Princeton, NJ, 1972).

[7] S.J. Perantonis and J.F. Wheater, Interacting fermions on a random lattice, Oxford University preprint (June 1987).

[8] D. Weingarten and B. Velikson, Nucl. Phys. B 270[FS16] (1986) 10; L. Jacobs, Phys. Rev. Lett. 51 ( 1983 ) 172.

[9] V. Alessandrini and A. Krzywicki, Phys. Lett. B 177 (1986) 395 ;

J.L. Alonso and J.L. Cortes, Phys. Lett. B 187 (1986) 146; M. Gross, P. Lepage and P.E.L. Rakow, Phys. Lett. B 197 (1987) 183.

[10] T.W. Chiu, Chiral fermions on the random-block lattice, in preparation. 